\section{PRELIMINARY ANALYSIS OF A REAL-WORLD STUDY (RWS) OF CAMRELIZUMAB TREATMENT IN PRIMARY LIVER CANCER (PLC)}

'Zhendong Chen, 'Nianfei Wang*, ${ }^{1}$ Dayong Luo, 'Bo Jiang, ${ }^{3}$ Mu Yuan, ${ }^{4}$ Xinzhong Li, ${ }^{5}$ Chunmei Yao, ${ }^{6}$ Hong Qian, ${ }^{7}$ Qingsheng Kan, ${ }^{8}$ Erxuan Wang. ${ }^{1}$ The second Hospital of Anhui Medical University, Hefei, China; ${ }^{2}$ The second people's Hospital of Fuyang City, Fuyang, China; ${ }^{3}$ The first affiliated Hospital of Bengbu Medical College, Bengbu, China; ${ }^{4}$ Huaibei people's Hospital, Huaibei, China; ${ }^{5}$ Huaibei miners General Hospital Group, Huaibei, China; ${ }^{6}$ The people's Liberation Army Navy Anqing Hospital, Anqing, China; ${ }^{7}$ Suzhou Municipal Hospital of Anhui Province, Suzhou, China; ${ }^{8}$ Wangjiang County people's Hospital, Anqing, China

Background Immune checkpoint inhibitors (ICIs) have revolutionized the landscape of PLC management at all evolutionary stages. ${ }^{1}$ As an anti-programmed cell death-1 (PD-1) antibody, camrelizumab monotherapy and in combination with apatinib, an anti-angiogenetic tyrosine kinase inhibitor of vascular endothelial growth factor receptor (VEGFR)-2, chemotherapy or locoregional therapy, have demonstrated their efficacy in advanced hepatocellular carcinoma (HCC). ${ }^{2} \quad 3 \quad 4 \quad 5$

Methods This prospective, open-label, multi-center, observational RWS was conducted to evaluate efficacy and safety of camrelizumab in treatment of PLC in clinical practice. Eligible patients were histopathologically or cytologically identified HCC or intrahepatic cholangiocarcinoma, who were going to receive camrelizumab treatment, with age $\geq 18$ ages, Eastern Cooperative Oncology Group performance status (ECOG PS) score of $0-2$ and Child-Pugh score $\leq 9$. Patients were treated at clinician discretion. Three hundred patients were planned to enroll, including advanced or peri-operative PLC. The primary endpoint was progress-free survival for advanced PLC, whose efficacy was available to analysis. Efficacy was assessed per Response Evaluation Criteria in Solid Tumors version 1.1. Results From March 29, 2020 to June 10, 2021, a total of 147 eligible patients of advanced PLC were enrolled and included in this interim analysis, with 128 (87.1\%) men, 130 (94.9\%) ECOG PS of 0-1, 139 (94.6\%) HCC, 74 (50.4\%) Barcelona Clinic Liver Cancer stage C, 98 (66.7\%) Child-Pugh $\mathrm{B}$, and $72(49.0 \%)$ with extrahepatic metastases, shown in table 1. Of the 147 patients, $45(30.1 \%)$ patients were treated with camrelizumab monotherapy, 79 (53.8\%) patients with combination with angiogenesis inhibitors, of which 55 (37.4\%) in combination with apatinib, $21(14.3 \%)$ patients with camrelizumab and chemotherapy. Patients, who had at least one efficacy assessment, were included in the efficacy analyses. Up to July 19,2021 , with a median follow time of 6.2 months, 132 patients were available for efficacy analyses. Patient disposition was shown in figure 1 . Objective response rate (ORR) and disease control rate (DCR) were 10\%/30.8\%/35.3\% and $75.0 \% / 86.5 \% / 70.6 \%$ in camrelizumab monotherapy/combined with apatinib/combined with chemotherapy, respectively. (table 2) The most common camrelizumab-treatment related adverse events (AEs) included reactive cutaneous capillary endothelial proliferation (RCCEP) (12, 8.2\%), ICI-induced pneumonia (2, $1.4 \%)$, enterocolitis (2, 1.4\%), and nephritis (1, 0.7\%), of which all these AEs recovered. Other AEs included increase of transaminase $(5,3.4 \%)$ and hypertension (4, 2.7\%). All AEs were 1-2 grade and no treatment-related death occurred.
Abstract 452 Table 1 Baseline characteristics

Baseline characteristics $(\mathrm{N}=147)$

Average age

$57.79 \pm 13.06$

Sex

Male

$128(87.1 \%)$

Female

$19(12.9 \%)$

ECOG PS score $\quad 0$

$60(40.8 \%)$

1

$73(49.7 \%)$

2

$14(9.5 \%)$

Histopathological HCC

$139(94.6 \%)$

features

ICC

$8(5.4 \%)$

BCLC stage

$\mathrm{UK}$

$24(16.3 \%)$

B

$49(33.3 \%)$

C

$74(50.4 \%)$

Child-Pugh

A

$49(33.3 \%)$

B

$98(66.7 \%)$

Data are n (\%). ECOG-PS, Eastern Cooperative Oncology Group Performance Status; HCC, hepatocellular carcinoma; ICC, intrahepatic cholangiocarcinoma; BCLC, Barcelona Clinic Liver Cancer; UK, unknown.

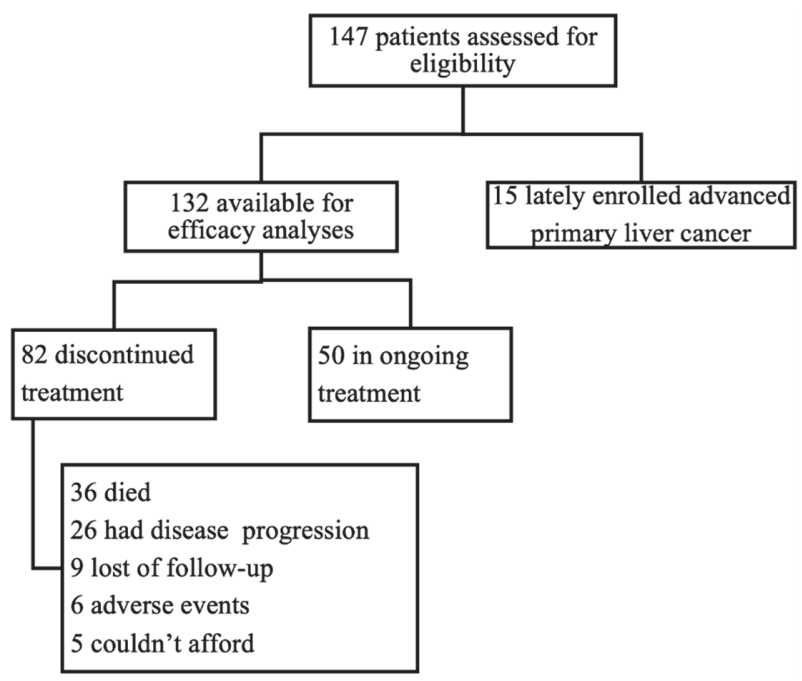

Abstract 452 Figure 1 Patient disposition 
Abstract 452 Table 2 Confirmed tumor response assessed by investigators per RECIST v1.1

\begin{tabular}{lcccccc}
\hline \multicolumn{6}{c}{ Confirmed tumor response assessed by investigators per } \\
RECIST v1.1
\end{tabular}

Conclusions Camrelizumab, combined with anti-angiogenetic agents or chemotherapy, or monotherapy, demonstrated good efficacy and safety in treatment of PLC.

Trial Registration ChiCTR2000034264

\section{REFERENCES}

1. Llovet JM, Kelley RK, Villanueva A, et al. Hepatocellular carcinoma. Nat Rev Dis Primers 2021;7(1):6-28.

2. Qin $S$, Ren $Z$, Meng Z, et al. Camrelizumab in patients with previously treated advanced hepatocellular carcinoma: a multicentre, open-label, parallel-group, randomised, phase 2 trial. Lancet Oncol 2020;21(4):571-580.

3. Xu J, Shen J, Gu S, et al. Camrelizumab in Combination with Apatinib in Patients with Advanced Hepatocellular Carcinoma (RESCUE): A Nonrandomized, Openlabel, Phase II Trial. Clin Cancer Res 2021;27(4):1003-1011.

4. Mei K, Qin $\mathrm{S}$, Chen Z, et al. Camrelizumab in combination with apatinib in second-line or above therapy for advanced primary liver cancer: cohort A report in a multicenter phase Ib/II trial. J Immunother Cancer 2021;9(3).

5. Qin S, Bai Y, Lim HY, et al. Randomized, multicenter, open-label study of oxaliplatin plus fluorouracil/leucovorin versus doxorubicin as palliative chemotherapy in patients with advanced hepatocellular carcinoma from Asia. J Clin Oncol 2013;31 (28):3501-3508.

Ethics Approval This study was approved by China registered clinical trial ethics review committee with No. ChiECRCT20200042.

http://dx.doi.org/10.1136/jitc-2021-SITC2021.452 